\title{
miR-203 inhibits arecoline-induced epithelial-mesenchymal transition by regulating secreted frizzled-related protein 4 and transmembrane-4 L six family member 1 in oral submucous fibrosis
}

\author{
LIAN ZHENG, XINCHUN JIAN, FENG GUO, NING LI, CANHUA JIANG, \\ PING YIN, AN-JIE MIN and LONG HUANG \\ Department of Oral and Maxillofacial Surgery, Xiangya Hospital of Central South University, \\ Changsha, Hunan 410008, P.R. China
}

Received February 17, 2015; Accepted March 16, 2015

DOI: $10.3892 /$ or.2015.3909

\begin{abstract}
Oral submucous fibrosis (OSF) is a potentially malignant disease predominantly found in Asian people. The areca nut has been implicated in this disease. Arecoline, one of the areca alkaloids, induces epithelial-mesenchymal transition (EMT)-related factors in primary human buccal mucosal fibroblasts. Yet, the mechanisms of the underlying arecoline-induced EMT in OSF remain unknown. In the present study, we aimed to investigate the role of microRNAs (miRNAs) in arecoline-induced EMT in HaCaT cells. We found that miR-203 was significantly downregulated in OSF tissues compared to that in normal buccal mucosa tissues, and that miR-203 negatively regulated secreted frizzled-related protein 4 (SFRP4) and positively regulated transmembrane-4 L six family member 1 (TM4SF1). We observed that upregulation of miR-203 significantly decreased the cell proliferation of $\mathrm{HaCaT}$ cells, and significantly upregulated the expression of cytokeratin 19 (CK19) and E-cadherin proteins, whereas it significantly downregulated the expression of $\mathrm{N}$-cadherin and vimentin compared to these levels in the vehicle control cells. Thus, we provide evidence to illustrate that miR-203 plays a role in the pathogenesis of OSF, which may be a target for OSF management.
\end{abstract}

\section{Introduction}

Oral submucous fibrosis (OSF) is a potentially malignant disease predominantly found in Asian people (1). OSF is

Correspondence to: Professor Xinchun Jian, Department of Oral and Maxillofacial Surgery, Xiangya Hospital of Central South University, 87 Xiangya Road, Changsha, Hunan 410008, P.R. China E-mail: jianxinchun@hotmail.com

Key words: oral submucous fibrosis, arecoline, epithelial-mesenchymal transition, microRNA, secreted frizzled-related protein, transmembrane-4 L six family member 1 characterized by submucosal fibrosis that affects most of the parts of the oral cavity and pharynx. OSF has been associated with oral squamous cell carcinoma, particularly in Taiwan and South-Central China where up to $80 \%$ of oral squamous cell carcinoma cases are associated with betel quid chewing (2). Possible etiological factors that have been implicated in this disease, include the areca nut, capsaicin in chillies, micronutrient deficiencies of iron, zinc and essential vitamins (3).

Arecoline, one of the areca alkaloids, is the main agent of the areca nut responsible for fibroblast proliferation (4). When influenced by slaked lime, arecoline is hydrolyzed to arecadine, which has pronounced effects on fibroblasts (5). It has been showed that arecoline exposure stimulates fibroblast growth (6). It was also reported that arecoline depleted cellular glutathione (GTH) levels, and subsequently induced various genotoxic and cytotoxic stimulation in oral mucosal fibroblasts (7). A recent study also demonstrated that arecoline induced epithelial-mesenchymal transition (EMT)-related factors in primary human buccal mucosal fibroblasts (6).

EMT is an indispensable mechanism during morphogenesis, and is also a crucial event in oral squamous cell carcinoma and OSF (8). Previous studies demonstrated that upregulation of several molecules involved in EMT, such as vimentin, were expressed in human buccal mucosal fibroblasts following arecoline treatment (9), suggesting that the EMT process may be directly involved in the pathogenesis of OSF. However, the mechanisms underlying the EMT induced by arecoline remain unknown.

MicroRNAs (miRNAs) are a class of short ( 22 nt) non-coding RNAs, which have been implicated in multiple cellular processes, including survival, proliferation, apoptosis and EMT in various types of cells (10). Recently, a study found that arecoline induced expression changes in miRNAs in normal mucosal cells (11). However, few studies have investigated the functions and the mechanism of differentially expressed miRNAs in OSF.

In the present study, we aimed to investigate the role of miRNAs in arecoline-induced EMT in HaCaT cells. Furthermore, we also explored the potential regulated targets 
of the miRNAs. We found that miR-203 was significantly downregulated in OSF tissues compared to that in normal buccal mucosa tissues, and that miR-203 negatively regulated secreted frizzled-related protein 4 (SFRP4), which is correlated with EMT-related gene expression (12), and positively regulated transmembrane-4 L six family member 1 (TM4SF1), a small plasma membrane glycoprotein that regulates cell motility and proliferation (13). Subsequently, we observed that upregulation of miR-203 significantly decreased the capacity of cell proliferation of HaCaT cells, and significantly upregulated the expression of cytokeratin 19 (CK19) and E-cadherin proteins, whereas it significantly downregulated the expression of $\mathrm{N}$-cadherin and vimentin compared to that of the vehicle control cells. Thus, we provide evidence to illustrate that miR-203 plays a role in the pathogenesis of OSF, which may be a target for OSF management.

\section{Materials and methods}

Sample collection. A total of 6 OSF tissue samples and 6 normal buccal mucosa tissues (Nor) were obtained from the Xiangya Hospital of Central South University according to the legislation and the Ethics Board of Xiangya Hospital. All subjects or their caregivers provided written informed consent. All samples were collected and identified by histopathological evaluation. All the samples were stored at $-80^{\circ} \mathrm{C}$ until being used.

Cell culture and treatment. HaCaT cells were purchased from ProCell Co. (Wuhan, China). All the cells were cultured in MEM supplemented with $15 \%$ fetal bovine serum in a $5 \% \mathrm{CO}_{2}$ humidified atmosphere at $37^{\circ} \mathrm{C}$. The cells were transfected with miR-203 mimics, miR-203 inhibitor, and a negative control (NC) using Lipofectamine 2000 (Invitrogen, Carlsbad, CA, USA) at a final concentration of $30 \mathrm{nM}$. To detect the effect of arecoline on EMT, the HaCaT cells were treated with arecoline (Selleck Chemicals, Houston, TX, USA) at the indicated concentrations for $72 \mathrm{~h}$.

Quantitative real-time polymerase chain reaction $(q P C R)$. The RNeasy Plus Mini kit (Qiagen, Valencia, CA, USA) was used to extract total RNA according to the manufacturer's instructions. The miRNeasy Mini kit (Qiagen) was used for real-time PCR to detect the expression of miR-203, miR30c and miR-206. The specific primer sets for miRNA-203, miR30c, miR-206 and U6 were purchased from GeneCopoeia. miR-203, miR30c and miR-206 expression was normalized to that of U6. The FastLane Cell SYBR ${ }^{\circledR}$-Green kit (Qiagen) was used for real-time PCR to detect the expression of TM4SF1 and SFRP4. The primers for TM4SF1, SFRP4 and GAPDH were: TM4SF1 sense, GCTGGAACAGGATGACTGCT and antisense, ACTCGGACCATGTGGAGGTA; SFRP4 sense, ATCTCGCCTGAAGCCATCG and antisense, GGGGCTTAG GCGTTTACAGT;GAPDH sense,CAATGACCCCTTCATTG ACC and antisense, GACAAGCTTCCCGTTCTCAG. TM4SF1 and SFRP4 expression was normalized to GAPDH. The $2^{-\Delta \Delta \mathrm{Ct}}$ method was used to analyze the data.

Western blotting. RIPA lysis buffer (Cwbiotech, Wuhan, China) was used to extract the total protein from the tissues and cells. The BCA protein assay kit (Thermo Fisher Scientific, Waltham, MA, USA) was used to measure the protein concentration. The total protein was separated by $10 \%$ SDS-PAGE and then transferred to nitrocellulose membranes. The membranes were blocked with $8 \%$ non-fat milk for $1 \mathrm{~h}$ and incubated with the indicated primary antibody (antiTM4SF1 and anti-SFRP4 from Epitomics; rabbit, 1:1,000; anti-N-cadherin and anti-vimentin from Abcam; rabbit, 1:500; anti-CK19, GAPDH and anti-E-cadherin from Santa Cruz; mouse, 1:500) overnight at $4^{\circ} \mathrm{C}$. The membranes were washed and incubated with the appropriate secondary antibody for $90 \mathrm{~min}$ at $37^{\circ} \mathrm{C}$. The signals on the membranes were detected by enhanced chemiluminescence (ECL) reagent. Data were analyzed by densitometry using Image-Pro Plus software 6.0 and normalized to internal control expression (GAPDH).

Dual luciferase reporter system. The wild-type 3'-UTR of TM4SF1 and SFRP4 was inserted into the dual luciferase reporter vector. For the luciferase assay, $10^{5}$ cells were plated and cultured in 24-well plates to reach $\sim 70 \%$ confluency. The cells were co-transfected with miR-203 mimics and the TM4SF1 or SFRP4 dual luciferase reporter vector, respectively. After a 48-h transfection, the Luciferase Reporter Gene Assay kit (Global Biotech, Shanghai, China) was used to determine the luciferase activitiy on a luminometer (Roche). Renilla luciferase activity was normalized to firefly luciferase activity.

CCK-8 cell proliferation assay. Cells $(1,000)$ were seeded in each well of 96 -well plates for $12 \mathrm{~h}$. Then, following the indicated treatment, the cells were further incubated for 0 , $12,24,48,72$ and $96 \mathrm{~h}$, respectively. CCK-8 reagent $(10 \mu \mathrm{l})$ (Dojindo, Tokyo, Japan) was added to the well at $1 \mathrm{~h}$ before the end of the incubation. The optical density (OD) value at $490 \mathrm{~nm}$ of each well was detected by an enzyme immunoassay analyzer.

Statistical analysis. Statistical analysis was performed by GraphPad Prism 5 and SPSS 16.0 softwares. The Student's t-test or one-way ANOVA was used depending on the experimental conditions. Data are expressed as mean $\pm \mathrm{SD}$. Compared to the controls, a P-value of $<0.05$ was considered to indicate a statistically significant result.

\section{Results}

miRNAs are differentially expressed in OSF tissues. We performed qPCR assay to detect the changes in miRNAs in 6 OSF tissues. Compared to the average expression in the normal oral mucosa tissues, the expression of miR-206 was significantly upregulated in 2 cases and slightly upregulated in 4 cases of OSF; the expression of miR-30c was significantly upregulated in 5 cases and slightly upregulated in 1 case of OSF; the expression of miR-203 was significantly upregulated in all of the 6 cases (Fig. 1A-C). We also detected the expression of potential target genes of these miRNAs by qPCR and western blotting in OSF tissues. We found that SFRP4 was increased in 5 of the 6 cases of OSF tissues at the mRNA level and upregulated in all the OSF tissues at the protein level compared with that in the normal tissues (Fig. 1D, F and G). 
A

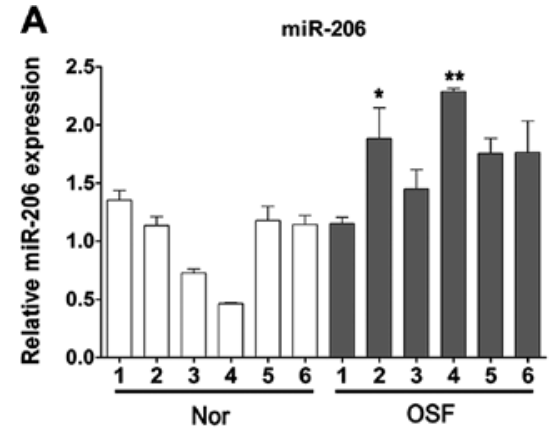

C

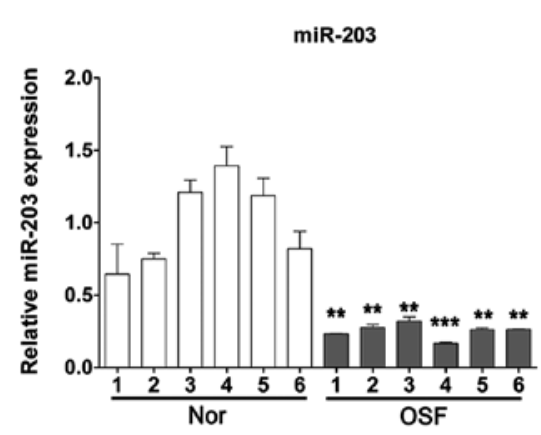

E

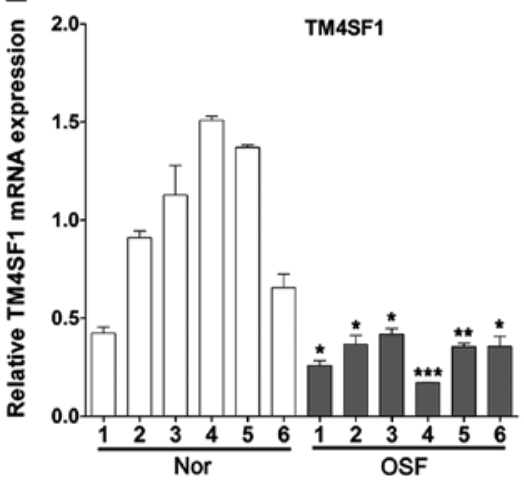

G

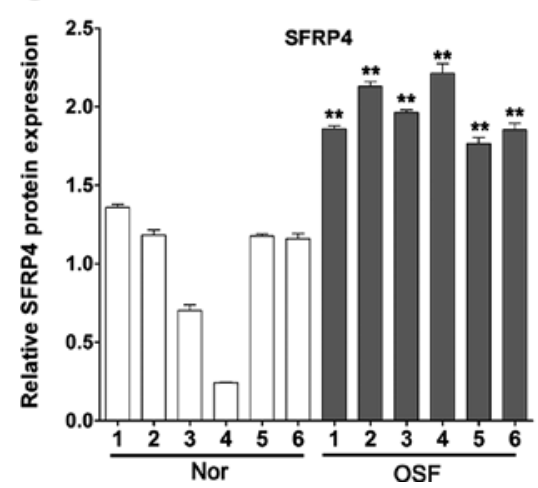

B $\quad$ miR-30c

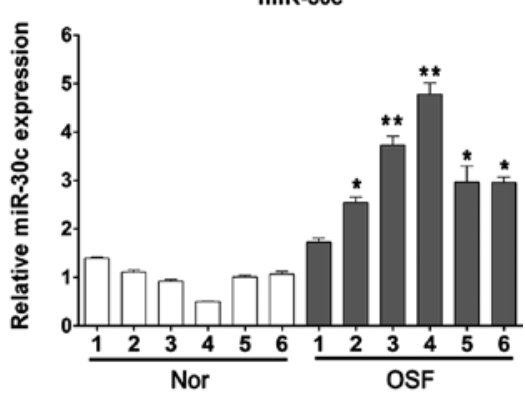

D

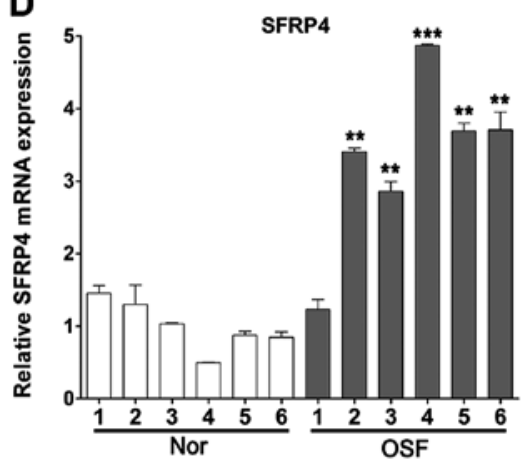

$\mathbf{F}$

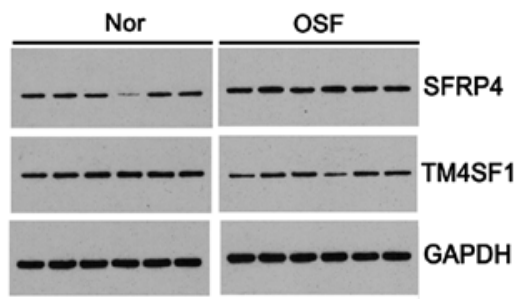

H

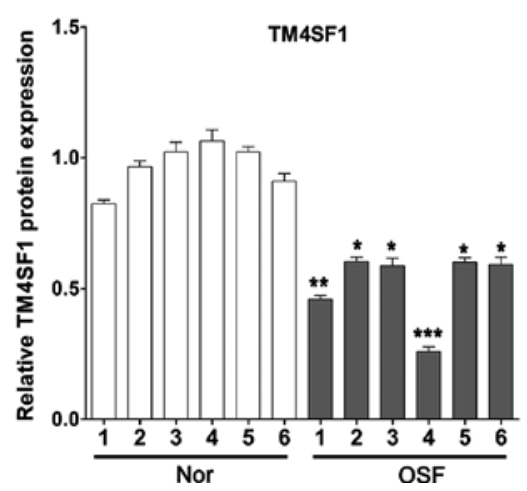

Figure 1. Expression of miR-206, miR-30c, miR-203, SFRP4 and TM4SF1 in OSF tissues and normal oral mucosa tissues. qPCR was utilized to detect the expression of (A) miR-206, (B) miR-30c, (C) miR-203, (D) SFRP4 and (E) TM4SF1 in OSF tissues and normal oral mucosa tissues. (F) Western blotting was used to analyze the expression of SFRP4 and TM4SF1 protein in OSF and normal oral mucosa tissues and quantification was carried out for (G) SFRP4 and (H) TM4SF1. Data are expressed as the means \pm SD. ${ }^{*} \mathrm{P}<0.05,{ }^{* *} \mathrm{P}<0.01,{ }^{* * *} \mathrm{P}<0.001$. SFRP4, secreted frizzled-related protein 4 ; TM4SF1, transmembrane-4 L six family member 1; OSF, oral submucous fibrosis.

In all of the 6 cases of OSF tissues and normal oral mucosa tissues, we found that TM4SF1 was significantly decreased at the mRNA and protein levels in the OSF tissues compared with levels in the normal tissues (Fig. 1E, F and H). 
A
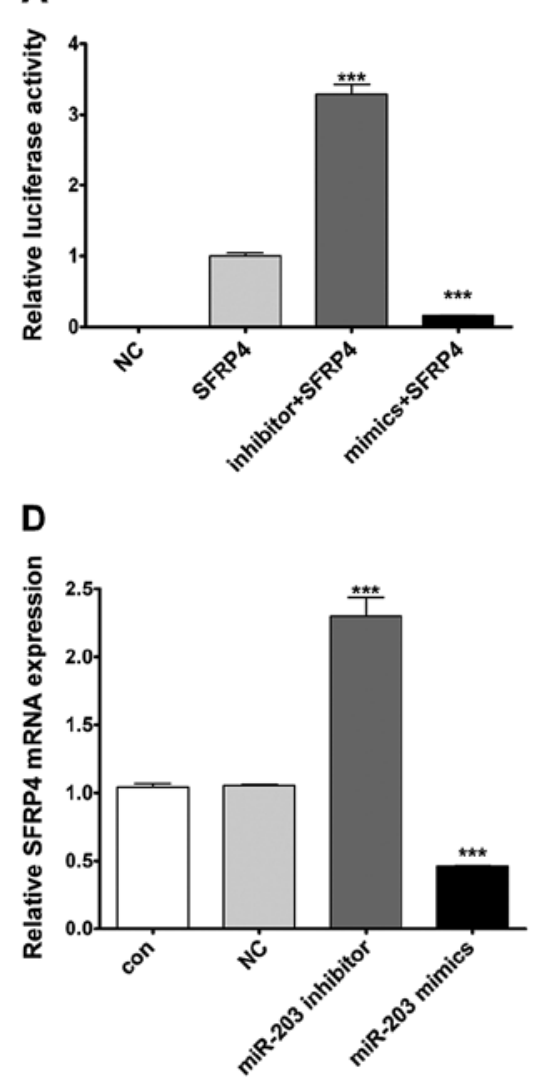

B
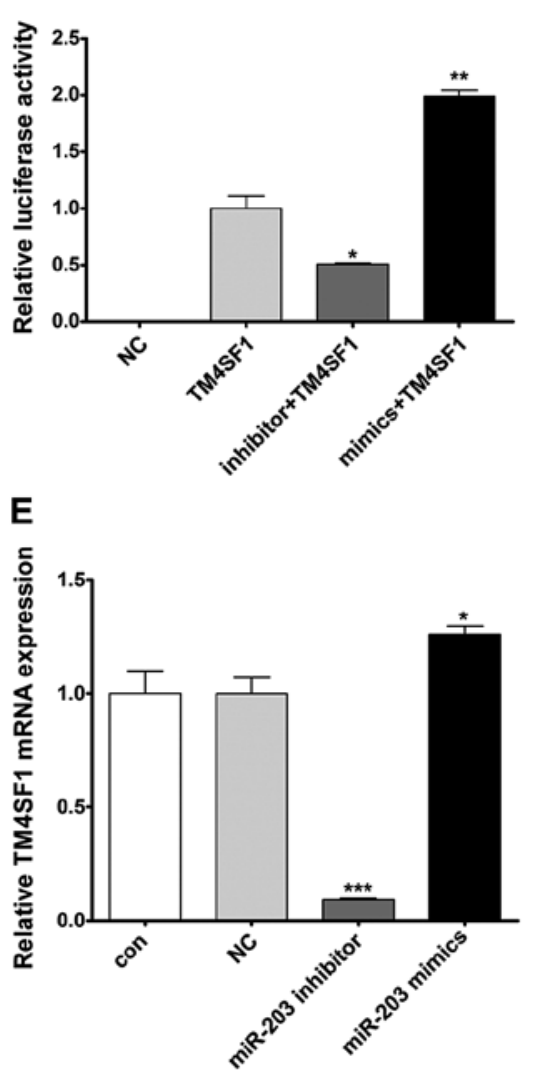

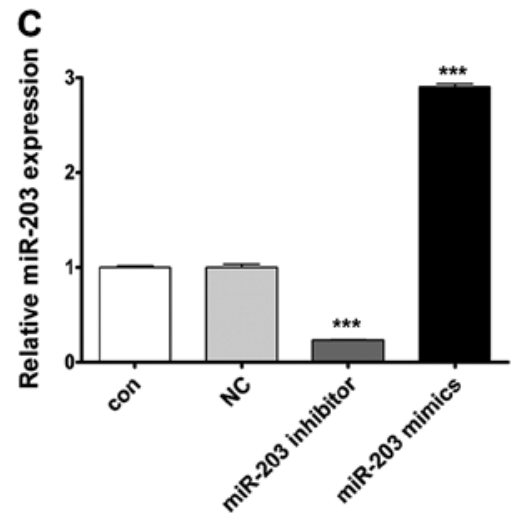

Figure 2. miR-203 negatively regulates SFRP4 and positively regulates TM4SF1. (A) The luciferase activity in cells transfected with SFRP4 3'UTR was decreased by the miR-203 mimics, whereas increased by miR-203 inhibitor. (B) The luciferase activity in cells transfected by TM4SF1 3'UTR was increased by miR-203 mimics, whereas decreased by miR-203 inhibitor. qPCR was used to detect the expression of (C) miR-203, (D) SFRP4 and (E) TM4SF1 mRNA after transfection with miR-203 mimic, miR-203 inhibitor or NC. Data shown are means $\pm \mathrm{SD},{ }^{*} \mathrm{P}<0.05,{ }^{* *} \mathrm{P}<0.01,{ }^{* * *} \mathrm{P}<0.001$. SFRP4, secreted frizzled-related protein 4; TM4SF1, transmembrane-4 L six family member 1.

miR-203 negatively regulates SFRP4 and positively regulates TM4SF1. To investigate whether miR-203 regulates the expression of SFRP4 and TM4SF1, we cloned the 3'UTR of SFRP4 and TM4SF1 downstream to the luciferase reporter gene. The constructed vectors were co-transfected with miR-203 mimics or inhibitor into the HaCaT cells. The luciferase activity of cells transfected with the miR-203 mimics and SFRP4 was significantly decreased compared with the cells that were transfected with SFRP4 alone, whereas the luciferase activity of cells transfected with miR-203 inhibitor and SFRP4 was significantly increased compared with the cells that were transfected with SFRP4 alone (Fig. 2A). In contrast to SFRP4, however, the luciferase activity of cells transfected with TM4SF1 was induced by co-transfection with the miR-203 mimics, while it was reduced by co-transfected with the miR-203 inhibitor (Fig. 2B). In order to further illustrate the regulatory relationship between miR-203 and SFRP4 and TM4SF1, we transfected the miR-203 mimics or inhibitor into $\mathrm{HaCaT}$ cells to upregulate or downregulate miR-203 expression. The efficiency of transfection was satisfied for further analysis (Fig. 2C). qPCR showed that enhanced or repressed miR-203 significantly decreased or increased SFRP4 mRNA levels, respectively, compared to the cells transfected with NC in the HaCaT cells (Fig. 2D). In addition, upregulation or downregulation of miR-203 significantly induced or reduced TM4SF1 mRNA levels, respectively, compared to the cells transfected with $\mathrm{NC}$ in the HaCaT cells (Fig. 2E). These results revealed that miR-203 negatively regulated SFRP4 and positively regulated TM4SF1 at the transcriptional levels.

Arecoline affects the expression of EMT-related genes in a dose-dependent manner. To determine the effects of arecoline on EMT in HaCaT cells, the HaCaT cells were treated with a series of increased doses of arecoline for $72 \mathrm{~h}$. Western blotting was used to evaluate the changes in expression of the EMT-related genes, including CK19, E-cadherin, N-cadherin and vimentin. The results showed that CK19 expression was significantly decreased with increased concentrations of arecoline; E-cadherin expression was markedly reduced at $0.08 \mathrm{mM}$; while the expression of $\mathrm{N}$-cadherin and vimentin was significantly upregulated with increasing concentrations of arecoline (Fig. 3). Downregulated expression of CK19 and E-cadherin, and upregulated expression of $\mathrm{N}$-cadherin and vimentin are important promotive factors of EMT. Thus, the concentration of arecoline at $0.08 \mathrm{mM}$ was chosen for further analysis.

Effects of arecoline on miR-203, SFRP4 and TM4SF1 in the HaCaT cells. To further analyze the effects of arecoline on 

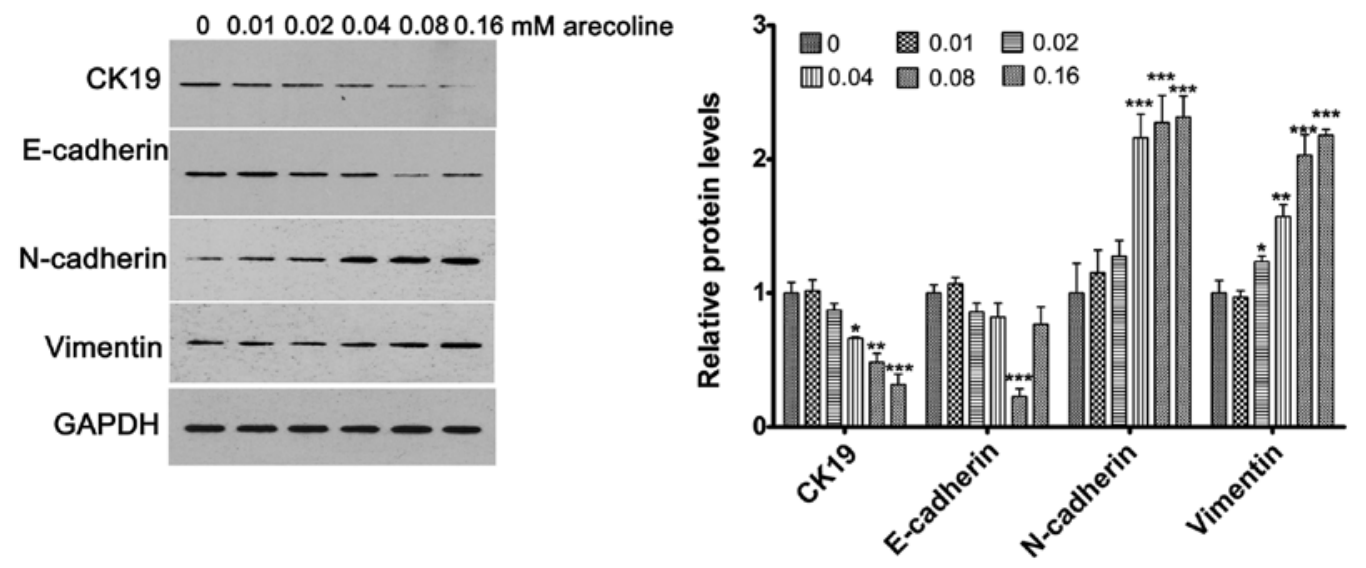

Figure 3. Arecoline affects expression of epithelial-mesenchymal transition-related genes in a dose-dependent manner. Expression of epithelial-mesenchymal transition-related genes (CK19, E-cadherin, $\mathrm{N}$-cadherin and vimentin) in $\mathrm{HaCaT}$ cells was determined by western blotting and the results were quantified. Data shown are means $\pm \mathrm{SD},{ }^{*} \mathrm{P}<0.05,{ }^{* *} \mathrm{P}<0.01,{ }^{* * *} \mathrm{P}<0.001$. CK19, cytokeratin 19.

A

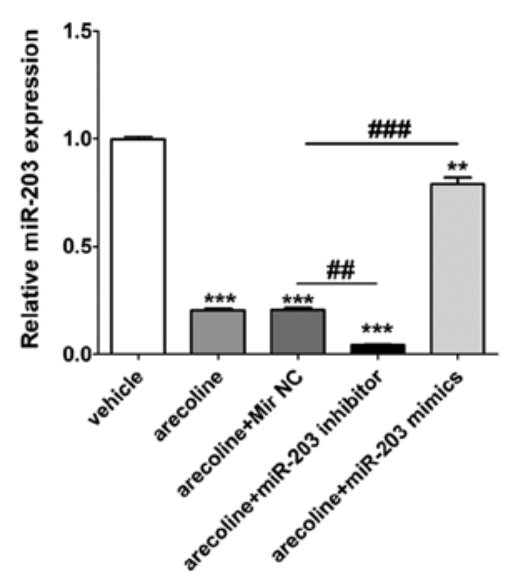

D

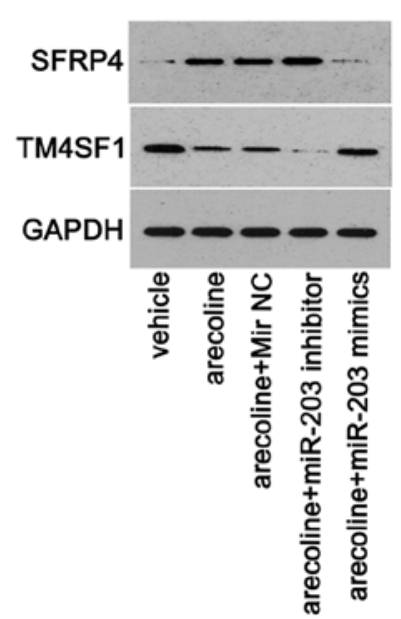

\section{B}
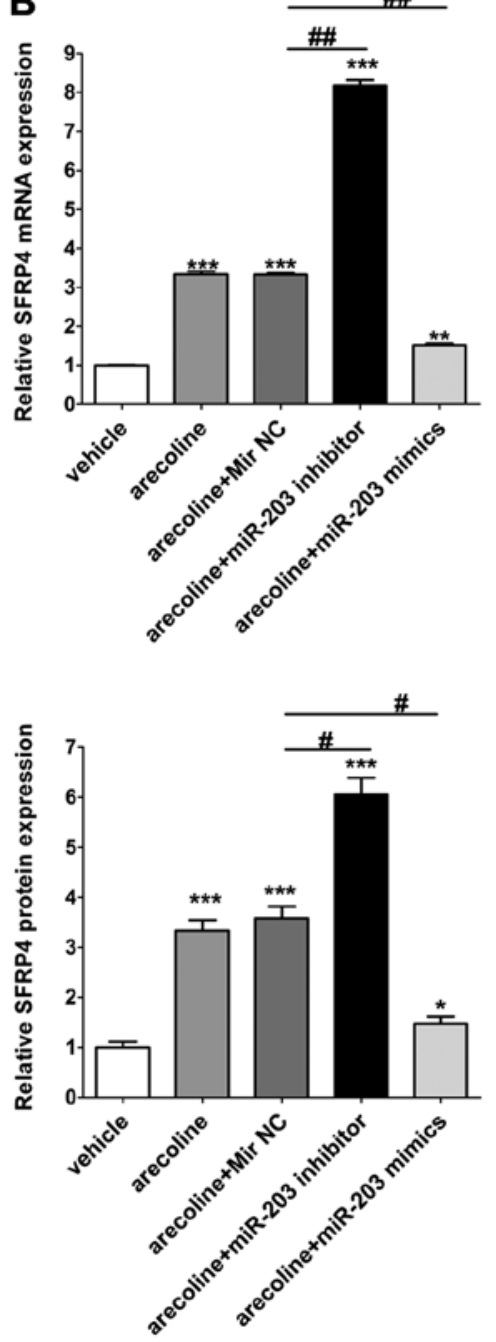
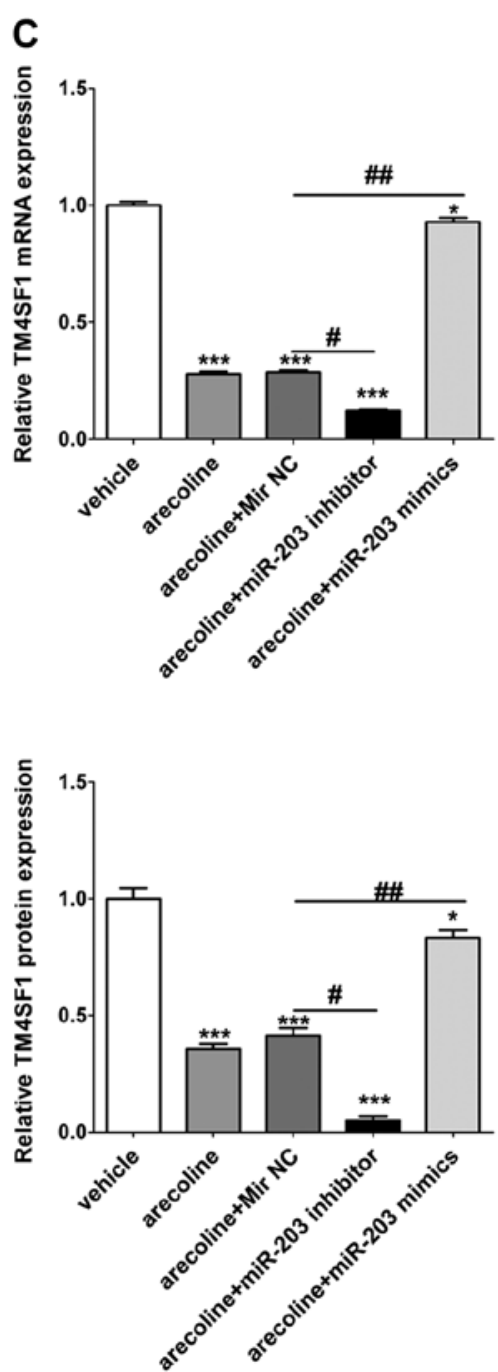

Figure 4. Effects of arecoline and miR-203 on SFRP4 and TM4SF1 in the HaCaT cells. qPCR was used to detect the expression of (A) miR-203, (B) SFRP4 mRNA and (C) TM4SF1 mRNA. (D) Western blotting was used to detect the expression of SFRP4 and TM4SF1 and the results were quantified. Data shown are means $\pm \mathrm{SD},{ }^{*} \mathrm{P}<0.05,{ }^{* *} \mathrm{P}<0.01,{ }^{* * *} \mathrm{P}<0.001 ;{ }^{\#} \mathrm{P}<0.05,{ }^{\# \#} \mathrm{P}<0.01,{ }^{\# \# \#} \mathrm{P}<0.001$. SFRP4, secreted frizzled-related protein 4; TM4SF1, transmembrane-4 L six family member 1 .

miR-203, SFRP4 and TM4SF1 in the HaCaT cells, cells were treated with arecoline alone or co-treated with miR-203 mimics/ inhibitor. We found that arecoline significantly decreased the expression of miR-203 compared with the vehicle control, 

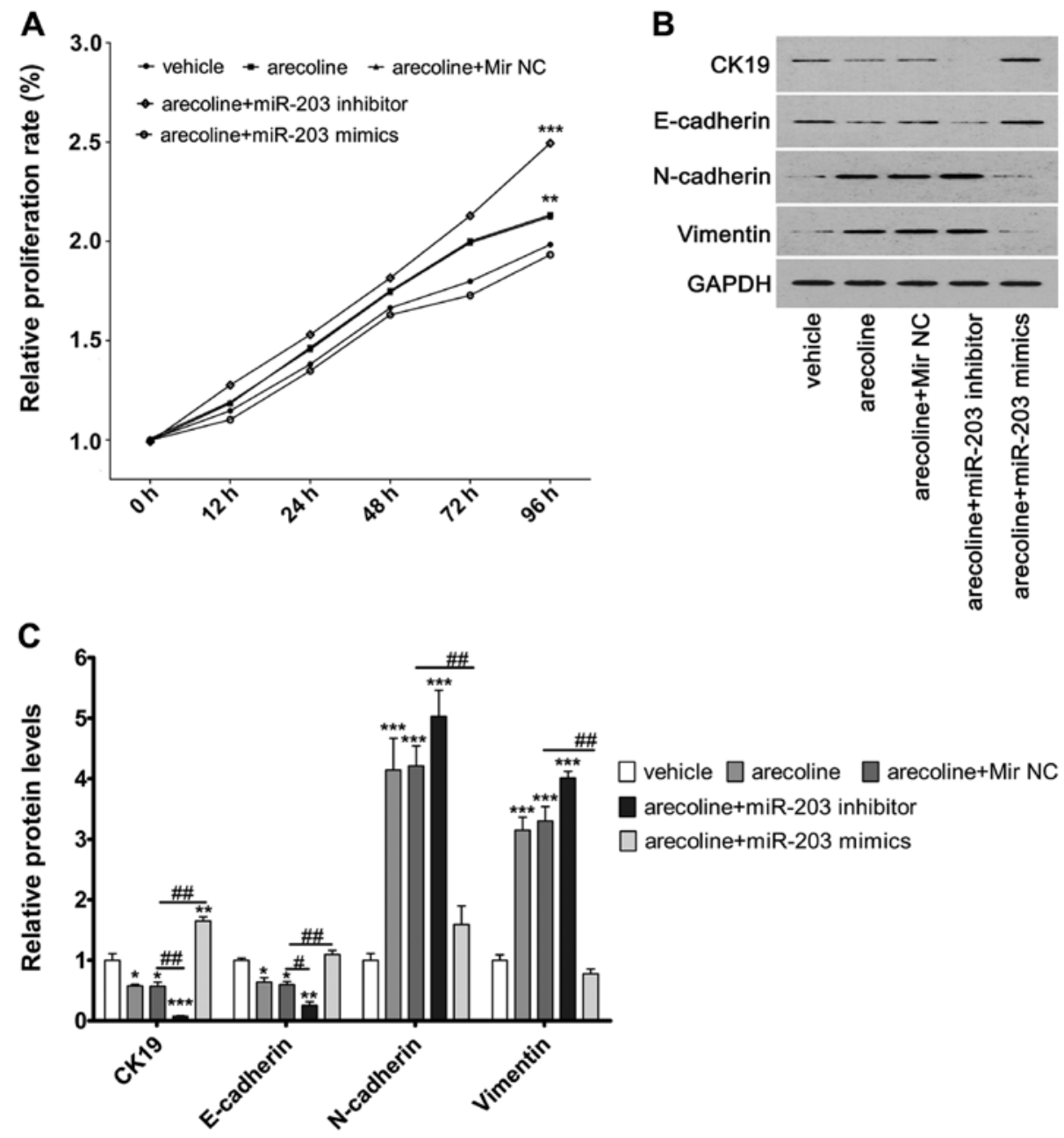

Figure 5. Effects of arecoline and miR-203 on HaCaT cells. (A) CCK-8 cell proliferation assay was used to evaluate cell proliferation. Data are expressed as $450 \mathrm{~nm}$ optical density in the different groups. (B and C) Expression of epithelial-mesenchymal transition-related genes (CK19, E-cadherin, N-cadherin and vimentin) in the $\mathrm{HaCaT}$ cells was determined by western blotting and quantified. Data shown are means $\pm \mathrm{SD},{ }^{*} \mathrm{P}<0.05,{ }^{* *} \mathrm{P}<0.01,{ }^{* * *} \mathrm{P}<0.001 ;{ }^{*} \mathrm{P}<0.05,{ }^{\# \#} \mathrm{P}<0.01$. CK19, cytokeratin 19.

and had a synergistic effect with the miR-203 inhibitor while co-treatment with miR-203 mimics rescued the expression of miR-203 that was decreased by arecoline (Fig. 4A). qPCR and western blotting were performed to detect the expression of SFRP4 and TM4SF1 in the HaCaT cells after the indicated treatments. We found that arecoline significantly increased the expression of SFRP4 compared with that in the vehicle control, and had a synergistic effect with the miR-203 inhibitor; while co-treatment with miR-203 mimics reversed the upregulated expression of SFRP4 that was induced by arecoline (Fig. 4B). Inversely, arecoline significantly reduced the expression of TM4SF1 compared with the vehicle control, and had a synergistic effect with the miR-203 inhibitor; while co-treatment with miR-203 mimics reversed the downregulated expression of TM4SF1 that was decreased by arecoline (Fig. 4C). The results of the western blotting further validated the phenomenon mentioned above at the translational level (Fig. 4D). These results indicated that arecoline affected the expression of miR-203, and subsequently regulated the expression of SFRP4 and TM4SF1.

miR-203 regulates cell proliferation, and regulates the expression of CK19, E-cadherin, $N$-cadherin and vimentin in the HaCaT cells. We observed that downregulation of miR-203 mediated by arecoline or co-treatment with the miR-203 inhibitor significantly increased cell proliferation ability in the HaCaT cells compared to the vehicle control cells. Furthermore, we observed that co-treatment of miR-203 mimics and arecoline significantly decreased the upregulation of cell proliferation induced by arecoline (Fig. 5A). This indicated that miR-203 regulated the proliferation of $\mathrm{HaCaT}$ cells.

To explore the potential molecular mechanisms underlying miR-203-induced cell proliferation and EMT, we assessed the expression of CK19, E-cadherin, N-cadherin and vimentin in $\mathrm{HaCaT}$ cells. Downregulation of miR-203 induced by arecoline or miR-203 inhibitor significantly downregulated the expression of CK19 and E-cadherin proteins compared to these levels in the vehicle control cells, whereas it significantly upregulated the expression of $\mathrm{N}$-cadherin and vimentin. Reversal of the downregulation of miR-203 in the arecolinetreated cells by miR-203 mimic transfection significantly upregulated the expression of CK19 and E-cadherin proteins compared to these levels in the vehicle control cells, whereas it significantly downregulated the expression of $\mathrm{N}$-cadherin and vimentin (Fig. 5B and C). 


\section{Discussion}

miRNAs have been implicated in inflammation, fibrosis, EMT and various types of cancers, such as oral cancer. Some miRNA families have gained attention for their clear function in tissue fibrosis. miR-30c has been reported to be a tumor suppressor in endometrial cancer (14) and to act as an independent predictor for the clinical benefit of tamoxifen therapy in patients with advanced breast cancer (15). A previous study showed that miR-206 overexpression inhibited estrogen receptor- $\alpha$-dependent proliferation, impaired invasiveness and induced cell cycle arrest of estrogen receptor- $\alpha$-positive endometrial endometrioid adenocarcinoma cell lines (16). miR-203 has previously been shown to play an important role in epithelial cell biology (17). miR-203 was differentially regulated in gingival epithelial cells in the presence of $P$. gingivalis. Silencing of miR-203 diminished the activation of signal transducer and activator of transcription 3 (Stat3), suggesting that alterations in miRNAs modulate important host signaling responses in pathological processes of severe periodontal disease (18). During master transcription factor-induced EMT in MCF7 breast cancer cells, miR-203 was repressed in a time-dependent manner. Dynamic simulations revealed stable epithelial and mesenchymal states, and underscored the crucial role of miR203 in state transitions underlying epithelial plasticity (19). Thus, according to these findings, we hypothesized that miR-203 plays a critical role in oral submucous fibrosis by regulating EMT-related genes via downstream target molecules.

In the present study, we found that the expression of miR-203 was significantly upregulated in all 6 OSF tissues, whereas upregulated expression of miR-206 and miR-30c was observed in 2 and 5 cases, respectively. Collectively, we therefore focused on the role of miR-203 in EMT of HaCaT cells for further analysis. We also found that SFRP4 was increased, while TM4SF1 was significantly decreased in OSF tissues at the mRNA and protein levels compared with that in the normal tissues. By dual luciferase report assay, we demonstrated that miR-203 negatively regulated SFRP4 and positively regulated TM4SF1 at the transcriptional levels. Secreted frizzled related protein (SFRP) proteins are characterized by a frizzled-like cysteine-rich domain and form a family of soluble proteins (SFRP1-5) (20). Upregulation of SFRP4 has been observed both in the skin of systemic sclerosis patients (21) and kidney fibrosis (22). Matsushima et al demonstrated that intramuscular administration of recombinant SFRP4 reduced fibrosis scar size and ameliorated cardiac function after ischemic injury (23). By oligonucleotide microarray that containing 15 cases of OSF tissues and 14 normal buccal mucosa tissues, our previously study found that expression of EMT-related gene SFRP4 was significantly upregulated in the OSF tissues compared with that in the normal mucosa tissues, which was validated by RT-PCR (24). This suggested that SFRP4 abnormalities in EMT may play an important role in the pathogenesis and malignant transformation of OSF. Transmembrane-4 L six family 1 (TM4SF1), the founding member of the L6 family, was originally identified as a protein abundantly expressed in a variety of epithelial cancer cells, and was found to share certain tetraspanin functions including roles in cell growth, motility and metastasis $(25,26)$. Previous findings indicated that TM4SF1 serves as a surface protein marker which identified mesenchymal stem cells from diverse cell sources, in particular, fibroblast-rich connective tissues (27). Thus, these data suggest that TM4SF1 is involved in the cell proliferation of epithelial cells during the processes of EMT. miRNAs regulate gene expression by inhibition of gene translation or facilitation of mRNA degradation. Our recent results showed that miR-203 directly targets the 3'UTR of SFRP4, and subsequently downregulated its expression. However, we also found that miR-203 upregulated TM4SF1 expression. We infer that miR-203 regulates the expression of TM4SF1 in an indirect manner, and further research should validate this.

CK19 is expressed exclusively by epithelial cells and derived cancers (28). Previous research indicated that CK19 expression may be implicated in the retention of proliferative potential or undifferentiated character in oral non-keratinized mucosa (29). We previously reported that the expression of CK19 at the mRNA or protein levels was shown to be significantly downregulated in the OSF basal cell layer, which is the proliferative invasive layer, than levels in normal buccal non-keratinized mucosa, indicating that the self-renewal capacity of the basal cell layer of OSF through stem cells was obviously inhibited (30). Similarly, stimulation by the areca nut also depressed the constant regeneration of OSF mucosa and promoted the atrophy of the oral epithelium. E-cadherin, a calcium-dependent cell-surface glycoprotein, is critical for maintaining epithelial cell-cell adhesion, cellular polarity differentiation, growth, cell migration and seems to be the most common target for various EMT signaling pathways (31). Downregulation of E-cadherin has been considered as a hallmark of EMT (32). During EMT, the epithelium expresses mesenchymal markers and becomes motile, and causes expression of N-cadherin which is present in mesenchymal cells (33). Enhanced expression of $\mathrm{N}$-cadherin in epithelial cells has been shown to decrease the endogenous levels of E-cadherin (34). Vimentin, one of the four types of intermediate filaments, is considered to play an important role in structural maintenance and adhesion in many cells originating from the mesenchymal (35). A previous immunohistochemical assay revealed that vimentin expression is significantly increased in OSF specimens than that in normal buccal mucosa. The upregulation of vimentin following arecoline exposure was consistent with that noted for fibroblasts cultured from OSF patients (9). By evaluating the changes in EMT hallmarks, CK19 and E-cadherin, and mesenchymal markers, $\mathrm{N}$-cadherin and vimentin, using western blotting, we validated that arecoline induced EMT in the HaCaT cells. We found that arecoline significantly decreased the expression of miR-203 and SFRP4, and increased the expression of TM4SF1. Furthermore, arecoline treatment markedly enhanced the cell growth of $\mathrm{HaCaT}$ cells. Importantly, we found that restoration of downregulated miR-203 induced by arecoline increased the expression of SFRP4, and decreased the expression of TM4SF1, and attenuated the cell proliferation of $\mathrm{HaCaT}$ cells. Moreover, the restoration of miR-203 expression in the arecoline-treated cells significantly upregulated the expression of CK19 and E-cadherin proteins, whereas it significantly downregulated the expression of $\mathrm{N}$-cadherin and vimentin. Our results suggest that miR-203 inhibits EMT by regulating the expression of SFRP4 and TM4SF1 in HaCaT cells. 
In conclusion, the present study provides evidence that miR-203 plays a critical role in arecoline-induced OSF by regulating the process of EMT, at least partially, via targeting SFRP4 and TM4SF1. Thus, miR-203 may be a target for the prevention and therapy of OSF.

\section{Acknowledgements}

This study was supported by the National Natural Sciences Foundation of China (no. 81260166, 81041052 and 30572044).

\section{References}

1. Lunde ML, Roman E, Warnakulasuriya S, Mehrotra R, Laranne J, Vasstrand EN and Ibrahim SO: Profiling of chromosomal changes in potentially malignant and malignant oral mucosal lesions from South and South-East Asia using array-comparative genomic hybridization. Cancer Genomics Proteomics 11: 127-140, 2014.

2. Hosthor SS, Mahesh P, Priya SA, Sharada P, Jyotsna M and Chitra S: Quantitative analysis of serum levels of trace elements in patients with oral submucous fibrosis and oral squamous cell carcinoma: A randomized cross-sectional study. J Oral Maxillofac Pathol 18: 46-51, 2014.

3. Mohammed F, Manohar V, Jose M, Fairozekhan Thapasum A, Mohamed S, Halima Shamaz B and D'Souza N: Estimation of copper in saliva and areca nut products and its correlation with histological grades of oral submucous fibrosis. J Oral Pathol Med 44: 208-213, 2014.

4. Zhou ZS, Li M, Gao F, Peng JY, Xiao HB, Dai LX, Lin SR, Zhang R and Jin LY: Arecoline suppresses HaCaT cell proliferation through cell cycle regulatory molecules. Oncol Rep 29: 2438-2444, 2013.

5. Wang TN, Huang MS, Lin MC, Duh TH, Lee CH, Wang CC, Chen PH, Chiang SL, Sheu CC, Chen VC, et al: Betel chewing and arecoline affects eotaxin-1, asthma and lung function. PLoS One 9: e91889, 2014.

6. Chang YC, Tsai CH, Lai YL, Yu CC, Chi WY, Li JJ and Chang WW: Arecoline-induced myofibroblast transdifferentiation from human buccal mucosal fibroblasts is mediated by ZEB1. J Cell Mol Med 18: 698-708, 2014.

7. Tsai CH, Yang SF, Chen YJ, Chu SC, Hsieh YS and Chang YC: Regulation of interleukin-6 expression by arecoline in human buccal mucosal fibroblasts is related to intracellular glutathione levels. Oral Dis 10: 360-364, 2004.

8. Yanjia H and Xinchun J: The role of epithelial-mesenchymal transition in oral squamous cell carcinoma and oral submucous fibrosis. Clin Chim Acta 383: 51-56, 2007.

9. Chang YC, Tsai CH, Tai KW, Yang SH, Chou MY and Lii CK: Elevated vimentin expression in buccal mucosal fibroblasts by arecoline in vitro as a possible pathogenesis for oral submucous fibrosis. Oral Oncol 38: 425-430, 2002.

10. Das RK, Anura A, Pal M, Bag S, Majumdar S, Barui A, Chakraborty C, Ray AK, Sengupta S, Paul RR, et al: Epitheliomesenchymal transitional attributes in oral sub-mucous fibrosis. Exp Mol Pathol 95: 259-269, 2013.

11. Liu B, Chen J and Jian X: Changes of miRNA after oral submucous fibrosis co-cultured with Salvia and low-dose prednisolone. Zhong Nan Da Xue Xue Bao Yi Xue Ban 39: 471-476, 2014 (In Chinese)

12. Ford CE, Jary E, Ma SS, Nixdorf S, Heinzelmann-Schwarz VA and Ward RL: The Wnt gatekeeper SFRP4 modulates EMT, cell migration and downstream Wnt signalling in serous ovarian cancer cells. PLoS One 8: e54362, 2013.

13. Lin CI, Merley A, Sciuto TE, Li D, Dvorak AM, MeleroMartin JM, Dvorak HF and Jaminet SC: TM4SF1: A new vascular therapeutic target in cancer. Angiogenesis 17: 897-907, 2014.

14. Kong X, Xu X, Yan Y, Guo F, Li J, Hu Y, Zhou H and Xun Q: Estrogen regulates the tumour suppressor MiRNA-30c and its target gene, MTA-1, in endometrial cancer. PLoS One 9: e90810, 2014.

15. Rodríguez-González FG, Sieuwerts AM, Smid M, Look MP, Meijer-van Gelder ME, de Weerd V, Sleijfer S, Martens JW and Foekens JA: MicroRNA-30c expression level is an independent predictor of clinical benefit of endocrine therapy in advanced estrogen receptor positive breast cancer. Breast Cancer Res Treat 127: 43-51, 2011.
16. Chen X, Yan Q, Li S, Zhou L, Yang H, Yang Y, Liu X and Wan X: Expression of the tumor suppressor miR-206 is associated with cellular proliferative inhibition and impairs invasion in ER $\alpha$-positive endometrioid adenocarcinoma. Cancer Lett 314: 41-53, 2012.

17. McKenna DJ, McDade SS, Patel D and McCance DJ: MicroRNA 203 expression in keratinocytes is dependent on regulation of p53 levels by E6. J Virol 84: 10644-10652, 2010.

18. Moffatt $\mathrm{CE}$ and Lamont RJ: Porphyromonas gingivalis induction of microRNA-203 expression controls suppressor of cytokine signaling 3 in gingival epithelial cells. Infect Immun 79: 2632-2637, 2011.

19. Moes M, Le Béchec A, Crespo I, Laurini C, Halavatyi A, Vetter G, Del Sol A and Friederich E: A novel network integrating a miRNA-203/SNAI1 feedback loop which regulates epithelial to mesenchymal transition. PLoS One 7: e35440, 2012.

20. Tendeng $\mathrm{C}$ and Houart $\mathrm{C}$ : Cloning and embryonic expression of five distinct $s f r p$ genes in the zebrafish Danio rerio. Gene Expr Patterns 6: 761-771, 2006

21. Bayle J, Fitch J, Jacobsen K, Kumar R, Lafyatis R and Lemaire R: Increased expression of Wnt 2 and SFRP4 in Tsk mouse skin: Role of Wnt signaling in altered dermal fibrillin deposition and systemic sclerosis. J Invest Dermatol 128: 871-881, 2008.

22. Surendran K, Schiavi S and Hruska KA: Wnt-dependent betacatenin signaling is activated after unilateral ureteral obstruction, and recombinant secreted frizzled-related protein 4 alters the progression of renal fibrosis. J Am Soc Nephrol 16: 2373-2384, 2005.

23. Matsushima K, Suyama T, Takenaka C, Nishishita N, Ikeda K, Ikada Y, Sawa Y, Jakt LM, Mori H and Kawamata S: Secreted frizzled related protein 4 reduces fibrosis scar size and ameliorates cardiac function after ischemic injury. Tissue Eng Part A 16: 3329-3341, 2010.

24. Hu Y, Jian X, Peng J, Jiang X, Li N and Zhou S: Gene expression profiling of oral submucous fibrosis using oligonucleotide microarray. Oncol Rep 20: 287-294, 2008.

25. Xu L, Li Q, Xu D, Wang Q, An Y, Du Q, Zhang J, Zhu Y and Miao Y: hsa-miR-141 downregulates TM4SF1 to inhibit pancreatic cancer cell invasion and migration. Int J Oncol 44: 459-466, 2014.

26. Tu SH, Huang HI, Lin SI, Liu HY, Sher YP, Chiang SK, Chong P, Roffler S, Tseng GC, Chen HW, et al: A novel HLA-A2-restricted CTL epitope of tumor-associated antigen L6 can inhibit tumor growth in vivo. J Immunother 35: 235-244, 2012.

27. Bae S, Shim SH, Park CW, Son HK, Lee HJ, Son JY, Jeon C and Kim H: Combined omics analysis identifies transmembrane 4 L6 family member 1 as a surface protein marker specific to human mesenchymal stem cells. Stem Cells Dev 20: 197-203, 2011.

28. Fuchs E, Tyner AL, Giudice GJ, Marchuk D, RayChaudhury A and Rosenberg M: The human keratin genes and their differential expression. Curr Top Dev Biol 22: 5-34, 1987.

29. Michel M, Török N, Godbout MJ, Lussier M, Gaudreau P, Royal A and Germain L: Keratin 19 as a biochemical marker of skin stem cells in vivo and in vitro: Keratin 19 expressing cells are differentially localized in function of anatomic sites, and their number varies with donor age and culture stage. J Cell Sci 109: 1017-1028, 1996.

30. Li N, Jian X, Hu Y, Xu C, Yao Z and Zhong X: Discovery of novel biomarkers in oral submucous fibrosis by microarray analysis. Cancer Epidemiol Biomarkers Prev 17: 2249-2259, 2008.

31. Thiery JP and Sleeman JP: Complex networks orchestrate epithelial-mesenchymal transitions. Nat Rev Mol Cell Biol 7: 131-142, 2006.

32. Wu CH, Tang SC, Wang PH, Lee H and Ko JL: Nickel-induced epithelial-mesenchymal transition by reactive oxygen species generation and E-cadherin promoter hypermethylation. J Biol Chem 287: 25292-25302, 2012.

33. Wheelock MJ, Shintani Y, Maeda M, Fukumoto $Y$ and Johnson KR: Cadherin switching. J Cell Sci 121: 727-735, 2008.

34. Islam S, Carey TE, Wolf GT, Wheelock MJ and Johnson KR: Expression of $\mathrm{N}$-cadherin by human squamous carcinoma cells induces a scattered fibroblastic phenotype with disrupted cell-cell adhesion. J Cell Biol 135: 1643-1654, 1996.

35. Guarino M, Tosoni A and Nebuloni M: Direct contribution of epithelium to organ fibrosis: Epithelial-mesenchymal transition. Hum Pathol 40: 1365-1376, 2009. 